\title{
Delayed Hypersensitivity Reactions Following First Dose of the SARS-CoV2 mRNA Vaccines
}

J Gen Intern Med 36(10):3298-300

DOI: $10.1007 / \mathrm{s} 11606-021-07015-w$

(c) Society of General Internal Medicine 2021

\section{BACKGROUND}

Vaccination is crucial to curtail the spread of SARS-CoV2; however, reports of adverse reactions including allergic reactions have increased public fears of the vaccine. ${ }^{1}$ The incidence of true hypersensitivity including both immediate and delayed reactions is estimated at $\sim 13$ cases per million. ${ }^{2}$ Delayed allergic reactions are heterogeneous, and there are limited reports regarding delayed reactions to the SARS-CoV2 mRNA vaccines.

\section{OBJECTIVE}

In this study, we characterize delayed hypersensitivity reactions to Pfizer/Moderna SARS-CoV2 mRNA vaccines at a single institution, and outcomes with second-dose administration.

\section{METHODS AND FINDINGS}

This was a retrospective study of delayed adverse reactions to the COVID-19 vaccine in healthcare workers at a single institution obtained through occupational health records. Patients included in the study experienced delayed reactions > $6 \mathrm{~h}$ following their first dose of Pfizer-BioNTech or Moderna SARS-CoV2 mRNA vaccine at vaccine clinics between 12/ $17 / 2020$ and 02/16/2021. Subjects were identified through a review of occupational health records that documented delayed reactions reported by employees via telephone. Patients with immediate reactions ( $\leq 6 \mathrm{~h}$ from vaccine administration) and known vaccine side effects were excluded.

Patients with delayed adverse reactions concerning true hypersensitivity were evaluated by an allergist and infectious disease specialist via in-person or e-consult visits. Based upon reaction severity, a recommendation was made for seconddose avoidance versus administration via full- or graded-dose challenge.

Received June 9, 2021

Accepted June 28, 2021

Published online July 21, 2021
A total of 138 reactions were reported out of 20,657 first doses of Pfizer and Moderna vaccines administered (0.66\%) during this period. One hundred nine patients were excluded due to immediate reactions and 5 patients were excluded due to known adverse reactions such as fever and fatigue. Of the 29 patients with delayed reactions, 14 patients were excluded due to symptoms that were incongruent with an underlying allergic mechanism. Ten of the 138 patients $(7.25 \%)$ were classified as having probable delayed onset hypersensitivity reaction to the first dose of vaccination (Table 1), with female predominance $(80 \%)$.

Delayed reactions included swelling around the eyes or face, systemic hives or rash, serum-sickness-like reaction, and large localized reaction. Two patients developed periorbital or perioral swelling within $24 \mathrm{~h}$ that rapidly resolved with antihistamines. Six patients described systemic hives or rash within $8 \mathrm{~h}$ to 4 days after vaccination that resolved spontaneously or following treatment with antihistamines and systemic steroids. One patient developed isolated large local reaction without systemic symptoms. The last subject experienced a serum-sickness-like reaction that developed approximately 7 days post-vaccination. Symptoms included urticaria, arthralgias, and myalgias, and labs demonstrated mild eosinophilia (350 cells/mcL), transaminitis (ALT 129/AST 212), elevated D-dimer (1302), and elevated CRP (21.8). COVID test was negative. Repeat labs 2 weeks later showed resolution of eosinophilia, transaminitis, and inflammatory markers.

Eight of the 10 patients elected to receive the second dose of vaccination and all 8 patients tolerated it without additional adverse events. Of the 8 patients, 6 patients received the second vaccine dose following standard protocol and 2 patients received the vaccine via graded-dose challenge, receiving $10 \%$ first followed by $90 \%$ the following week based on the time course of the index reaction. Two of the 10 patients elected not to receive the second dose of vaccine.

\section{DISCUSSION}

Although immediate type 1 anaphylactic reactions to vaccinations are most concerning, any vaccine reactions can dissuade patients from receiving their second dose to be fully vaccinated. ${ }^{3}$ In our cohort, common delayed reactions to vaccination included hives/rash, swelling/angioedema, and localized reactions. These reactions were largely self-limited and managed 


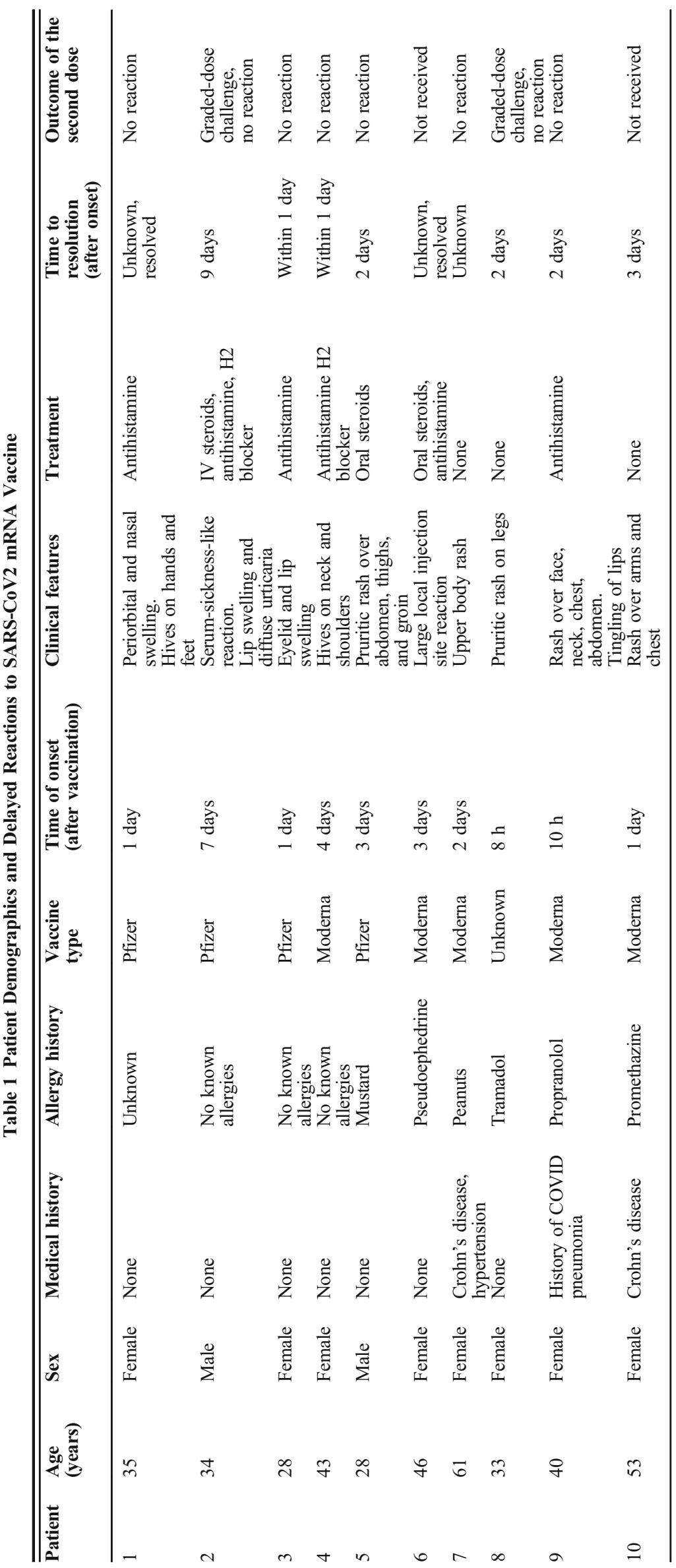


with antihistamines, oral steroids, or self-resolved, similar to previously described delayed vaccine reactions. ${ }^{3}$ Large localized vaccine reactions have been attributed to a T-cellmediated response and are not a contraindication to the second dose. ${ }^{4}$ Interestingly, one patient experienced a serumsickness-like reaction that traditionally prohibits re-challenge. However, following recovery, the patient elected to receive his second vaccine dose without issues.

The limitations of this study were that it was retrospective and relied on subject self-reporting. Despite temporal correlation with the reaction, the vaccine may or may not have been causal.

Our study characterizes delayed hypersensitivity reactions and outcomes with second-dose administration. Patientreported delayed reactions did not preclude tolerance of subsequent vaccine doses in any of the patients who elected to receive their second vaccine dose. Understanding the mechanism and implication of immune-mediated vaccine reactions is essential for maintaining this intervention.

Jennifer $\mathrm{Xu}, M D^{1}$

Kristine Vanijcharoenkarn, $M D^{2}$

Mary Elizabeth Sexton, $M D^{3}$

Lindsay Martin, FNP-BC ${ }^{4}$

Frances Eun-Hyung Lee, $M D^{2}$

Merin Elizabeth Kuruvilla, $M D^{2}$

${ }^{1}$ Department of Medicine, Emory University School of Medicine,

Atlanta, GA, USA
${ }^{2}$ Division of Pulmonary, Allergy and Critical Care, Emory University School of Medicine, Atlanta, GA, USA

${ }^{3}$ Division of Infectious Disease, Emory University School of Medicine,

Atlanta, GA, USA

${ }^{4}$ Department of Employee Health, Emory University School of Medicine,

Atlanta, GA, USA

Corresponding Author: Merin Elizabeth Kuruvilla, MD; Division of Pulmonary, Allergy and Critical Care, Emory University School of Medicine, Atlanta, GA, USA (e-mail: merin.kuruvilla@emoryhealthcare.org).

DECLARATIONS:

CONFLICT OF INTEREST: The authors declare that they do not have a conflict of interest.

\section{REFERENCES}

1. COVID Data Tracker. 2021 4/20/21]; Available from: https://covid-cdcgov.proxy.library.emory.edu/covid-data-tracker/\#datatracker-home.

2. Allergic Reactions Including Anaphylaxis After Receipt of the First Dose of Moderna COVID-19 Vaccine - United States, December 21, 2020-January 10, 2021. MMWR Morb Mortal Wkly Rep, 2021. 70(4): p. 125-129.

3. McNeil, M.M. and F. DeStefano, Vaccine-associated hypersensitivity. J Allergy Clin Immunol, 2018. 141(2): p. 463-472.

4. Blumenthal, K.G., et al., Delayed Large Local Reactions to mRNA-1273 Vaccine against SARS-CoV-2. N Engl J Med, 2021. 384(13): p. 1273-1277.

Publisher's Note: Springer Nature remains neutral with regard to jurisdictional claims in published maps and institutional affiliations. 